\title{
РАЗРЕШИМОСТЬ КРАЕВОЙ ЗАДАЧИ ДЛЯ \\ РАЗНОПОРЯДКОВОГО ДИФФЕРЕНЦИАЛЬНОГО УРАВНЕНИЯ НА ГЕОМЕТРИЧЕСКОМ ГРАФЕ
}

\section{К. П. Лазарев, Т. В. Белоглазова}

В работе исследуется разрешимость краевой задачи для струнно-стержневой модели. Модель описывается уравнением, которое имеет второй или четвертый порядок на различных ребрах произвольного графа. Получены критерии вырожденности и невырожденности задачи; в частности, доказано, что невырожденность задачи эквивалентна принципу максимума.

Библиография: 22 названия.

В различных разделах естествознания и техники возникают модели, описываемые дифференциальными уравнениями на геометрических графах (сетях). Исследованию таких задач посвящены сотни работ, часть из которых приведены в [1], [2]. Основные продвижения сделаны в изучении обыкновенных дифференциальных уравнений второго порядка на графах (см., например, [1], [3]-[8]). Для обыкновенных дифференциальных уравнений четвертого порядка на графах в [9][14] исследовались асимптотика спектра и проблемы управляемости, в [1], [15]-[18] были рассмотрены краевые задачи для моделей стержневых систем с различными условиями шарнирного или упруго-шарнирного сочленения, получены условия разрешимости и свойства функции Грина (симметричность, непрерывность, гладкость, положительность). Разнопорядковые задачи на графах пока исследованы мало. В этом направлении известны результаты Ю. В. Покорного и К. П. Лазарева [19], [20] для модели растянутой цепочки из струн и стержней, Ю. В. Покорного и Е. Н. Провоторовой [8], [21] для модели пучка из одинаковых струн и одинаковых стержней. В работе [22] рассмотрена разнопорядковая задача для модели "стержневой треугольник, растянутый за вершины струнами" и анонсированы ее разрешимость, существование непрерывной и неотрицательной функции Грина. В настоящей работе исследуется задача для струнно-стержневой модели, описанной уравнением, которое имеет второй или четвертый порядок на различных ребрах произвольного графа. Приведены критерии вырожденности и невырожденности задачи и показано, что невырожденность задачи эквивалентна выполнению принципа максимума.

Работа выполнена при поддержке грантов Президента РФ по поддержке ведущих научных школ № НШ-1643.2003.1, Российского фонда фундаментальных исследований, грант № 04-01-00049, Минобразования РФ (КЦ СПбГУ, проект № Е02-1.0-46) и программы “Университеты России", проект УР.04.01.004. 
1. Основные понятия и постановка задачи. Введем необходимые понятия из теории краевых задач на графах, следуя в основном [1], [5], [16].

Пусть в $\mathbb{R}^{3}$ задано множество попарно непересекающихся одномерных интервалов $\gamma_{i}, i=\overline{1, r}$, называемых ребрами, и множество их концов, называемых вершинами. Взаимное расположение ребер и вершин определяется отношением примыкания, которое, с одной стороны, указывает, какие вершины соединяются данным ребром, а с другой - какие ребра примыкают к данной вершине. Предполагается, что к каждой вершине примыкает, по крайней мере, одно ребро и каждое ребро соединяет две некоторые вершины. Если ребро $\gamma$ соединяет вершины $a$ и $b$, то мы будем писать $\gamma=(a, b)$.

Геометрическим графом Г будем называть объединение всех ребер $\gamma_{i}$ и некоторых вершин, которые мы будем называть внутренними. Вершины, не вошедшие в Г, называются граничными. Множество ребер $\left\{\gamma_{1}, \ldots, \gamma_{r}\right\}$ графа Г будем обозначать через $E(\Gamma)$, множество вершин - через $V(\Gamma)$, множество внутренних вершин - через $J(\Gamma)$, а множество граничных вершин - через $\partial \Gamma$. Для каждой вершины $a \in V(\Gamma)$ обозначим через $\Gamma(a)$ множество всех ребер, примыкающих к $a$.

Введем на графе индуцированную из $\mathbb{R}^{3}$ топологию. Граф называется связным, если его нельзя представить в виде объединения двух непустых непересекающихся открыто-замкнутых подмножеств. Обозначим $\Gamma^{0}=\bigcup_{i=1}^{r} \gamma_{i}$ (объединение точек всех ребер), $\bar{\Gamma}=\Gamma \cup \partial \Gamma$ (замыкание графа). Подграф графа $\Gamma$ - это граф $\Lambda$ такой, что $\Lambda$ является открытым подмножеством $Г$ и $J(\Lambda) \subset J(\Gamma)^{1}$.

Выделим подграфы $\Gamma_{1}$ и $\Gamma_{2}$, образованные ребрами из $E(\Gamma)$ так, что $J\left(\Gamma_{1}\right) \cap$ $J\left(\Gamma_{2}\right)=\varnothing$ и $E\left(\Gamma_{1}\right) \cup E\left(\Gamma_{2}\right)=E(\Gamma)$.

Введем необходимые классы функций.

Функция на графе - это вещественнозначная функция, заданная на $\Gamma^{0}$. Сужение функции на графе $u(\cdot)$ на произвольное множество $A$ обозначим через $u_{A}(\cdot)$.

Если сужение функции на графе на каждое ребро $\gamma \in E(\Gamma)$ имеет производную по параметру $t$ при его натуральной параметризации $x=\varphi_{\gamma}(t)$, то будем говорить, что функция имеет производную на графе, обозначаемую через $u^{\prime}(x)$. Таким образом, в точке $x \in \gamma$ имеем $u^{\prime}(x)=\frac{d}{d t} u\left(\varphi_{\gamma}(t)\right)$. Аналогично определяются производные $u^{(k)}(\cdot), \quad k \geqslant 2$. При изменении направления параметризации на ребре знак производной на этом ребре меняется на противоположный. Ясно, что производные (или квазипроизводные) нечетного порядка зависят от выбора направления параметризации ребер, а четного порядка - нет. Ниже будет рассмотрено разнопорядковое дифференциальное уравнение на графе, имеющее второй или четвертый порядок на ребрах и, следовательно, инвариантное относительно направления параметризации ребер.

Пусть $C(\gamma)$ для $\gamma \in E(\Gamma)$ - это множество функций $u: \gamma \rightarrow \mathbb{R}$, равномерно непрерывных на $\gamma$. Аналогично, $C^{k}(\gamma)$ - это множество функций, равномерно непрерывных вместе с производными до порядка $k$.

Введем пространства

$$
C\left(\Gamma^{0}\right)=\left\{u(\cdot) \mid u: \Gamma^{0} \longrightarrow \mathbb{R}, u_{\gamma} \in C(\gamma), \gamma \in E(\Gamma)\right\}
$$

\footnotetext{
1 Заметим, что некоторые вершины из $\partial \Lambda$ могут быть внутренними точками ребер графа Г, и тогда ребро графа $\Lambda$, примыкающее к такой вершине, является частью ребра графа $Г$.
} 


$$
\begin{aligned}
C^{k}\left(\Gamma_{i}^{0}\right) & =\left\{u(\cdot) \mid u: \Gamma_{i}^{0} \longrightarrow \mathbb{R}, u_{\gamma} \in C^{k}(\gamma), \gamma \in E\left(\Gamma_{i}\right)\right\}, \quad i=\overline{1,2}, \\
\mathscr{T} & =\left\{u(\cdot) \mid u \in C\left(\Gamma^{0}\right), u_{\Gamma_{1}^{0}} \in C^{4}\left(\Gamma_{1}^{0}\right), u_{\Gamma_{2}^{0}} \in C^{2}\left(\Gamma_{2}^{0}\right)\right\}
\end{aligned}
$$

Заметим, что функция $u(\cdot)$ из пространства $\mathscr{T}$ имеет пределы в произвольной вершине графа вдоль каждого примыкающего к ней ребра. Эти пределы, вообе говоря, различны. Предел в вершине $a$ вдоль примыкающего к ней ребра $\gamma=(a, b)$ будем обозначать через $u_{\gamma}(a)$. При изучении краевой задачи мы будем продолжать функцию $u_{\gamma}(\cdot)$ значениями $u_{\gamma}(a)$ и $u_{\gamma}(b)$ и тогда на замыкании ребра $\bar{\gamma}$ продолженная функция (за ней сохраним то же самое обозначение) будет иметь производные в вершинах и принадлежать пространству $C^{k}(\bar{\gamma})$, где $k=4$ при $\gamma \in E\left(\Gamma_{1}\right)$ и $k=2$ при $\gamma \in E\left(\Gamma_{2}\right)$. Ниже при формулировании условий, содержащих производные в вершине $a$, нам удобнее пользоваться производными по направлению “от вершины", которые будем обозначать через $u_{\gamma}^{(k)}(a)$.

Пусть заданы функции $p \in C^{2}\left(\Gamma_{1}^{0}\right)$ и $q \in C^{1}\left(\Gamma_{2}^{0}\right)$, причем $\inf _{x \in \Gamma_{1}^{0}}\{p(x)\}>0$ и $\inf _{x \in \Gamma_{2}^{0}}\{q(x)\}>0$.

При $f \in C\left(\Gamma^{0}\right)$ рассмотрим краевую задачу на графе $\Gamma$

$$
\begin{aligned}
\left(p_{\gamma} u_{\gamma}^{\prime \prime}\right)^{\prime \prime} & =f_{\gamma}(x), \quad x \in \gamma, \quad \gamma \in E\left(\Gamma_{1}\right), \\
-\left(q_{\gamma} u_{\gamma}^{\prime}\right)^{\prime} & =f_{\gamma}(x), \quad x \in \gamma, \quad \gamma \in E\left(\Gamma_{2}\right), \\
u_{\gamma}(a) & =u_{\mu}(a), \quad \gamma, \mu \in \Gamma(a), \quad a \in J(\Gamma), \\
\left(p_{\gamma} u_{\gamma}^{\prime \prime}\right)(a) & =0, \quad \gamma \in \Gamma_{1}(a), \quad a \in V(\Gamma), \\
\sum_{\gamma \in \Gamma_{1}(a)}\left(p_{\gamma} u_{\gamma}^{\prime \prime}\right)^{\prime}(a) & =\sum_{\gamma \in \Gamma_{2}(a)}\left(q_{\gamma} u_{\gamma}^{\prime}\right)(a), \quad a \in J(\Gamma), \\
u_{\gamma}(a) & =0, \quad \gamma \in \Gamma(a), \quad a \in \partial \Gamma .
\end{aligned}
$$

Здесь при $\Gamma_{1}(a)=\varnothing$ отсутствуют условия (4) в вершине $a$ и первая сумма в условии (5) равна нулю. Аналогично, при $\Gamma_{2}(a)=\varnothing$ вторая сумма в условии (5) равна нулю. При $\partial \Gamma=\varnothing$ отсутствуют условия (6).

Решением задачи (1)-(6) называем функцию $u \in \mathscr{T}$, сужения которой на ребра $\gamma \in E\left(\Gamma_{1}\right)$ (соответственно $\gamma \in E\left(\Gamma_{2}\right)$ ) всюду удовлетворяют уравнениям (1) (соответственно (2)), и для которой выполнены условия (3)-(6).

Задачу (1)-(6) будем называть невырожденной, если соответствующая однородная задача имеет только нулевое решение в $\mathscr{T}$.

Очевидно, что задача (1)-(6) однозначно разрешима при любой правой части $f \in C\left(\Gamma^{0}\right)$ тогда и только тогда, когда она невырождена.

Введем разнопорядковый дифференциальный оператор $L: \mathscr{T} \longrightarrow C\left(\Gamma^{0}\right)$, который действует на функции $u \in \mathscr{T}$ по правилу $(L u)(x)=\left(p u^{\prime \prime}\right)^{\prime \prime}(x)$ при $x \in \Gamma_{1}^{0}$, $(L u)(x)=-\left(q u^{\prime}\right)^{\prime}(x)$ при $x \in \Gamma_{2}^{0}$. Оператор $\mathfrak{L}$ определим на функциях $u \in \mathscr{T}$, удовлетворяющих условиям (3)-(5), формулой $\mathfrak{L} u=L u$.

Будем говорить, что для уравнения $\mathfrak{L} u=0$ выполнен приниип максимума, если для любого решения $u(\cdot)$ этого уравнения $\sup _{\Gamma} u(\cdot)$ и $\inf _{\Gamma} u(\cdot)$ достигаются на $\partial Г$, т.е. существуют $a \in \partial \Gamma, \gamma \in \Gamma(a), \quad b \in \partial \Gamma, \quad \mu \in \Gamma(b)$ такие, что $\sup _{\Gamma} u(\cdot)=u_{\gamma}(a)$ и $\inf _{\Gamma} u(\cdot)=u_{\mu}(b)$. Здесь максимум и минимум могут быть нестрогими и достигаться на целых ребрах, примыкающих к граничным вершинам. 
В п. 2 исследуются условия вырожденности и невырожденности задачи (1)-(6), в частности, показано, что принцип максимума эквивалентен невырожденности задачи.

2. Невырожденность краевой задачи. Пусть $m_{i}=\left|E\left(\Gamma_{i}\right)\right|^{2}, \quad i=\overline{1,2}$ и $m=$ $4 \cdot m_{1}+2 \cdot m_{2}$. Легко видеть, что решение однородного уравнения $L u=0$ определяется $m$ параметрами.

Подсчитаем число независимых условий (3)-(6). Условия (4) дают удвоенное количество ребер из $E\left(\Gamma_{1}\right)$, примыкающих ко всем вершинам, так как каждое ребро примыкает к двум вершинам. Число условий (6) равно числу ребер из $E(\Gamma)$, примыкающих к граничным вершинам. Число независимых условий (3) в вершине $a$ на единицу меньше числа ребер, примыкающих к $a$, поэтому число независимых условий (3) и (5) равно числу ребер из $E(\Gamma)$, примыкающих к внутренним вершинам. Таким образом, общее число независимых условий (3)-(6) также равно $m$ и формально задача (1)-(6) является определенной.

Для графа $\Gamma$, содержащего одно ребро $\gamma=(a, b)$, задача невырождена, если, по крайней мере, одна из вершин является граничной; в противном случае задача вырождена, так как нетривиальным решением однородной задачи является постоянная.

Действительно, при $m_{1}=0, m_{2}=1$ имеем уравнение $-\left(q u^{\prime}\right)^{\prime}=0$ на $\gamma$. Для $a, b \in \partial \Gamma$ выполнены условия $u(a)=u(b)=0$, а для $a \in \partial \Gamma, b \in J(\Gamma)-$ условия $u(a)=$ $-\left(q u^{\prime}\right)(b)=0$ и, значит, задача невырождена. Если же обе вершины внутренние, то выполнены условия $-\left(q u^{\prime}\right)(a)=-\left(q u^{\prime}\right)(b)=0$ и, следовательно, задача вырождена.

Аналогично, при $m_{1}=1, m_{2}=0$ имеем уравнение $\left(p u^{\prime \prime}\right)^{\prime \prime}=0$ на $\gamma$. Для $a, b \in \partial \Gamma$ выполнены условия $u(a)=\left(p u^{\prime \prime}\right)(a)=0, u(b)=\left(p u^{\prime \prime}\right)(b)=0$, а для $a \in \partial \Gamma, b \in J(\Gamma)-$ условия $u(a)=\left(p u^{\prime \prime}\right)(a)=0,\left(p u^{\prime \prime}\right)(b)=\left(p u^{\prime \prime}\right)^{\prime}(b)=0$ и, значит, задача невырождена. Если же обе вершины внутренние, то выполнены условия $\left(p u^{\prime \prime}\right)(a)=\left(p u^{\prime \prime}\right)^{\prime}(a)=0$, $\left(p u^{\prime \prime}\right)(b)=\left(p u^{\prime \prime}\right)^{\prime}(b)=0$ и, следовательно, задача вырождена.

Для произвольного графа ниже в теореме 1 приведены условия вырожденности, в теореме 2 приведены условия невырожденности, а в теореме 3 показано, что выполнение условия теоремы 1 равносильно невыполнению условия теоремы 2 и, таким образом, получены необходимые и достаточные условия вырожденности и невырожденности задачи.

ТЕОРема 1. Задача (1)-(6) вырождена, если существует связный подграф $\Lambda$ графа Г такой, что

а) $\Lambda$ содержит только целье ребра графа $\Gamma$;

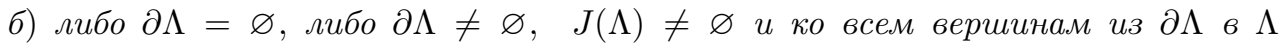
примыкают только ребра $\gamma \in E\left(\Gamma_{1}\right)$ (на этих ребрах порядок оператора $L$ равен четырем).

ДокАЗАТЕЛьСтво. Пусть $\partial \Lambda=\varnothing$. В этом случае имеем $V(\Lambda)=J(\Lambda) \subset J(\Gamma)$ и, следовательно, в вершинах графа $\Lambda$ отсутствуют условия (6). Тогда функция $u(x)=1$ на $\Lambda$ и $u(x)=0$ на $\Gamma \backslash \Lambda$ является нетривиальным решением однородной задачи.

Рассмотрим случай $\partial \Lambda \neq \varnothing$. Покажем сначала, что если $\gamma=(a, b)-$ произвольное ребро графа $\Lambda$, примыкающее к вершине $a \in \partial \Lambda$, то $b \in J(\Lambda)$. Для графа $\Lambda$,

\footnotetext{
2 Через $|A|$ обозначено число элементов конечного множества $A$.
} 
состоящего из одного ребра, $b \in J(\Lambda)$ в силу условия б). Пусть теперь $|E(\Lambda)|>1$. Если $b \notin J(\Lambda)$, то $b \in \partial \Lambda$. Следовательно, вершины $a$ и $b$ не принадлежат $\Lambda$. Тогда $\Lambda=\gamma \cup(\Lambda \backslash \gamma)$, причем $\gamma \cap(\Lambda \backslash \gamma)=\varnothing$ и $\gamma, \Lambda \backslash \gamma$ - непустые открыто-замкнутые подмножества $\Lambda$. А это противоречит связности $\Lambda$. Итак, $b \in J(\Lambda)$.

Теперь построим нетривиальное решение однородной задачи. Естественно искать решение в виде линейной функции на ребрах из $\Gamma_{1}$ и постоянной на ребрах из $\Gamma_{2}$. Положим $u(x) \equiv 0$ вне $\Lambda$ и в граничных вершинах $\partial Г$. Для каждого ребра $\gamma=$ $(a, b)$ подграфа $\Lambda$, примыкающего к произвольной вершине $a \in \partial \Lambda$, положим $u_{\gamma}(x)$ линейной и такой, что $u_{\gamma}(a)=0$ и $u_{\gamma}(b)=1$. При этом по условию теоремы $\gamma \in$ $E\left(\Gamma_{1}\right)$ и по доказанному $b \in J(\Lambda)$. На всех остальных ребрах из $\Lambda$ возьмем $u(x) \equiv 1$. Очевидно, $u \in \mathscr{T}$.

Покажем, что построенная функция $u(x)$ удовлетворяет однородной задаче. На тех ребрах, где $u(x) \equiv 0$ или $u(x) \equiv 1$, имеем $u^{\prime}(x) \equiv 0$ и $L u(x) \equiv 0$. Остальные ребра примыкают к граничным вершинам из $\partial \Lambda$ и принадлежат $E\left(\Gamma_{1}\right) \cap E(\Lambda)$. На этих ребрах $u(x)$ линейна; тогда $\left(p u^{\prime \prime}\right)(x) \equiv 0$ и $L u(x) \equiv 0$. Итак, уравнение $L u=0$ выполнено.

Проверим выполнение условий (3)-(6). Во-первых, условия (3) выполняются, так как в каждой вершине $a \in J(\Lambda)$ имеем $u_{\gamma}(a)=1$ для всех ребер $\gamma \in \Gamma(a)$, а в каждой вершине $a \notin J(\Lambda)$ имеем $u_{\gamma}(a)=0$ для всех ребер $\gamma \in \Gamma(a)$. Во-вторых, условия (4) выполнены, так как $u(\cdot)$ линейна на всех ребрах графа $\Gamma_{1}$. В-третьих, условия (5) выполнены. Действительно, на любом ребре осуществляется одна из трех ситуаций: $u(x) \equiv 0, u(x) \equiv 1, u(x)$ линейна и принимает значения между нулем и единицей, причем в последнем случае ребро принадлежит $E\left(\Gamma_{1}\right)$. Поэтому все слагаемые в $(5)$ нулевые. В-четвертых, условия (6) выполнены по построению.

Итак, задача (1)-(6) вырождена и теорема доказана.

Теорема 2. Задача (1)-(6) невырождена, если $J(\Gamma)=\varnothing$ или $J(\Gamma) \neq \varnothing, \partial \Gamma \neq \varnothing$

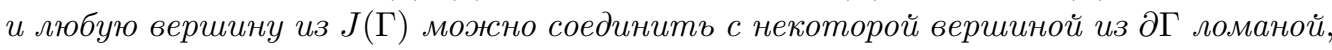
звенъя которой совпадают с ребрами графа $\Gamma_{2}$ (на этих ребрах порядок оператора $L$ равен двум).

ДокАЗАТЕЛЬСТво. Пусть $u(x)$ - решение однородной задачи. Используя условия $u \in \mathscr{T},(3)$ и $(6)$ продолжим $u(x)$ до непрерывной функции на $\bar{\Gamma}$.

Обозначим через $M$ значение $\max _{x \in \bar{\Gamma}} u(x)$. Покажем, что значение $M$ достигается в некоторой точке из $\partial \Gamma$ и $M=0$.

Предположим, что $u\left(x_{0}\right)=M$. При $x_{0} \in \partial \Gamma$ значение $M$ достигается на $\partial Г$. Если $x_{0}$ является внутренней точкой ребра $\gamma \in E\left(\Gamma_{2}\right)$, то $u^{\prime}\left(x_{0}\right)=0$ и в силу уравнения $\left(q u^{\prime}\right)^{\prime}=0$ имеем $\left(q u^{\prime}\right)(x) \equiv 0$ на $\gamma$. Отсюда следует, что $u^{\prime}(x) \equiv 0$ и $u(x) \equiv M$ на ребре $\gamma$ и в вершинах, примыкающих к нему. Если $x_{0}$ является внутренней точкой ребра $\gamma \in E\left(\Gamma_{1}\right)$, то также имеем $u^{\prime}\left(x_{0}\right)=0$. В силу уравнения $\left(p u^{\prime \prime}\right)^{\prime \prime}=0$ на $\gamma$ функция $p u^{\prime \prime}$ линейна и на концах ребра $\gamma$ в силу условий (4) обращается в нуль. Следовательно, $\left(p u^{\prime \prime}\right)(x) \equiv 0$ на $\gamma$. Отсюда и из условия $u^{\prime}\left(x_{0}\right)=0$ получаем $u^{\prime}(x) \equiv 0$ на $\gamma$. Поэтому $u(x) \equiv M$ на $\gamma$ и в вершинах, примыкающих к нему.

В случае графа без внутренних вершин из проведенных рассуждений следует, что значение $M$ достигается в некоторой точке из $\partial \Gamma$. 
Теперь рассмотрим случай, когда $x_{0}$ является внутренней вершиной графа Г. Из условий (5) имеем

$$
\sum_{\gamma \in \Gamma_{1}\left(x_{0}\right)}\left(p_{\gamma} u_{\gamma}^{\prime \prime}\right)^{\prime}\left(x_{0}\right)=\sum_{\gamma \in \Gamma_{2}\left(x_{0}\right)}\left(q_{\gamma} u_{\gamma}^{\prime}\right)\left(x_{0}\right),
$$

причем по условию теоремы $\Gamma_{2}\left(x_{0}\right) \neq \varnothing$. Если $\Gamma_{1}\left(x_{0}\right)=\varnothing$, то справедливо равенство $\sum_{\gamma \in \Gamma_{2}\left(x_{0}\right)}\left(q_{\gamma} u_{\gamma}^{\prime}\right)\left(x_{0}\right)=0$. Если же $\Gamma_{1}\left(x_{0}\right) \neq \varnothing$, то для всех $\gamma \in E\left(\Gamma_{1}\right)$ (и, в частности, для $\left.\gamma \in \Gamma_{1}\left(x_{0}\right)\right)$ из проведенных выше рассуждений следует, что $\left(p_{\gamma} u_{\gamma}^{\prime \prime}\right)(x) \equiv 0$, и тогда $\left(p_{\gamma} u_{\gamma}^{\prime \prime}\right)^{\prime}(x) \equiv 0$. Значит, $\sum_{\gamma \in \Gamma_{1}\left(x_{0}\right)}\left(p_{\gamma} u_{\gamma}^{\prime \prime}\right)^{\prime}\left(x_{0}\right)=0$ и также имеем

$$
\sum_{\gamma \in \Gamma_{2}\left(x_{0}\right)}\left(q_{\gamma} u_{\gamma}^{\prime}\right)\left(x_{0}\right)=0 .
$$

Поскольку $x_{0}$ - точка максимума, то $u_{\gamma}^{\prime}\left(x_{0}\right) \leqslant 0$ при каждом $\gamma \in \Gamma\left(x_{0}\right)$. Следовательно, в сумме $\sum_{\gamma \in \Gamma_{2}\left(x_{0}\right)}\left(q_{\gamma} u_{\gamma}^{\prime}\right)\left(x_{0}\right)$ все слагаемые неположительны. Поэтому $\left(q_{\gamma} u_{\gamma}^{\prime}\right)\left(x_{0}\right)=0$ при всех $\gamma \in \Gamma_{2}\left(x_{0}\right)$. Отсюда и из уравнения $\left(q u^{\prime}\right)^{\prime}(x)=0$, как и ранее, получаем $u(x) \equiv M$ на всех ребрах $\gamma \in \Gamma_{2}\left(x_{0}\right)$ и прилежащих к ним вершинам. По условию теоремы вершину $x_{0}$ можно соединить с некоторой вершиной $b \in \partial \Gamma$ ломаной, звенья которой совпадают с ребрами графа $\Gamma_{2}$, причем можно считать, что все узлы ломаной кроме $b$ являются внутренними вершинами графа Г. Тогда из проведенного рассуждения следует, что $u(x) \equiv M$ на этой ломаной и в граничной вершине $b$.

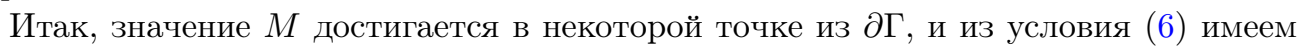
$M=0$.

Таким образом, $\max _{x \in \bar{\Gamma}} u(x)=0$ и, аналогично, $\min _{x \in \bar{\Gamma}} u(x)=0$. Следовательно, $u(x) \equiv 0$ на $\Gamma$, и задача $(1)-(6)$ невырождена.

Теорема доказана.

ТЕОРема 3. Для графа Г следующие условия эквивалентны:

$1^{0}$. существует вершина $а \in J(\Gamma)$, которую нелвзя соединить с дГ ломаной, звенъя которой совпадают с ребрами графа $\Gamma_{2}$;

$2^{0}$. существует связный подграф $\Lambda$ графа $Г$ такой, что выполнены условия а) и б) теоремы 1.

ДокАзАТЕЛЬСтво. Пусть сначала выполнено условие $1^{0}$. Граф $\Lambda$ составим из некоторых ребер и вершин графа Г. Если к вершине а примыкают только ребра графа $\Gamma_{1}$, то множество $J(\Lambda)$ составим из вершины $a$, множество $E(\Lambda)$ составим из всех ребер графа $\Gamma_{1}$, примыкающих к вершине $a$.

Если к вершине $а$ примыкают ребра не только графа $\Gamma_{1}$, то множество $J(\Lambda)$ составим из $а$ и тех внутренних вершин графа $\Gamma$, которые можно соединить с $a$ ломаной со звеньями, совпадающими с ребрами графа $\Gamma_{2}$, не примыкающими к $\partial Г$; множество $E(\Lambda)$ составим из всех ребер графа $\Gamma$, примыкающих к вершинам из $J(\Lambda)$.

Пусть $\Lambda^{0}-$ множество точек всех ребер из $E(\Lambda)$. Образуем множество $\Lambda=$ $J(\Lambda) \cup \Lambda^{0}$.

По построению множество $\Lambda$ составлено из целых ребер графа $\Gamma$, входящих в $E(\Lambda)$, и некоторых концов этих ребер, включенных в $J(\Lambda)$. Следовательно, $\Lambda$ является графом, у которого $J(\Lambda)$ - множество внутренних вершин, а $E(\Lambda)$ - множество 
ребер. В силу построения $\Lambda \subset \Gamma$ и $J(\Lambda) \subset J(\Gamma)$. Покажем, что $\Lambda$ - открытое подмножество в $Г$. Ребро графа является открытым подмножеством и, следовательно, любая точка ребра входит в $\Lambda$ с некоторой окрестностью. Если $b \in J(\Lambda)$, то $b \in J(\Gamma)$ и по построению все ребра графа $\Gamma$, примыкающие к вершине $b$, включены в $\Lambda$. Тогда $b$ вместе с примыкающими ребрами образует открытое подмножество точки $b$, которая целиком входит в $\Lambda$. Поэтому $\Lambda$ является открытым подмножеством и, следовательно, подграфом графа $Г$.

Покажем, что $\Lambda$ - связный подграф. Любую вершину $b \in J(\Lambda)$, отличную от $a$, можно соединить с вершиной $а$ ломаной со звеньями, совпадающими с ребрами из $\Gamma_{2}$ так, что эта ломаная включена в $\Lambda$. Далее, две произвольные внутренние вершины из $\Lambda$, отличные от $a$, можно соединить с вершиной $a$ и, следовательно, между собой ломаными такого же типа. Две произвольные точки в $\Lambda$ можно соединить отрезками ребер с вершинами из $J(\Lambda)$, затем эти вершины, как указано выше, соединить ломаной между собой и получим непрерывную кривую в $\Lambda$, соединяющую заданные точки. Таким образом, множество $\Lambda$ линейно связно и, следовательно, связно.

Условие а) выполнено по построению графа $\Lambda$.

Проверим выполнение условия б). При $\partial \Lambda=\varnothing$ условие б) выполнено. При $\partial \Lambda \neq \varnothing$ покажем, что к любой вершине $b \in \partial \Lambda$ в $\Lambda$ не могут примыкать ребра из $E\left(\Gamma_{2}\right)$. Предположим противное: в $\Lambda$ ребро $\gamma=(b, c)$ принадлежит $E\left(\Gamma_{2}\right)$. Тогда вершина $c$ по построению графа $\Lambda$ является его внутренней вершиной. Поскольку вершины $b$ и $c$ соединены ребром из $E\left(\Gamma_{2}\right)$, то $b \in J(\Lambda)$, что противоречит выбору вершины $b$. Следовательно, выполнены условие б) и вместе с ним $2^{0}$.

Пусть теперь выполнено условие $2^{0}$. Если $\partial \Gamma=\varnothing$, то условие $1^{0}$ выполнено. Рассмотрим случай $\partial \Gamma \neq \varnothing$. При выполнении условия б) любую вершину из $J(\Lambda)$ нельзя соединить с $\partial \Lambda$ ломаной, звенья которой совпадают с ребрами графа $\Gamma_{2}$.

Покажем, что любую вершину из $J(\Lambda)$ нельзя соединить с $\partial Г$ ломаной, звенья которой совпадают с ребрами графа $\Gamma_{2}$. Пусть, в предположении противного, вершины $a \in J(\Lambda)$ и $b \in \partial \Gamma$ соединены ломаной $\sigma$ со звеньями, совпадающими с ребрами графа $\Gamma_{2}$. Как отмечено выше, $b \notin \partial \Lambda$. Кроме того, в силу $J(\Lambda) \subset J(\Gamma)$ и $J(\Gamma) \cap \partial \Gamma=\varnothing$ имеем $b \notin J(\Lambda)$. Таким образом, $b$ не является вершиной графа $\Lambda$. Введем ориентацию на $\sigma$ от $a \mathrm{k} b$. Заметим, что первое звено ломаной $\sigma$ образовано ребром графа $\Lambda$, а последнее звено - ребром, не входящим в $\Lambda$. Поскольку $\Lambda-$ открытое подмножество в $\Gamma$, то любое ребро графа $\Lambda$, прилежащее к его внутренней вершине, включено в $\Lambda$. Поэтому существует набор идущих подряд начальных звеньев ломаной $\sigma$, которые входят в $\Lambda$. Последняя вершина $c$ этого набора ребер является граничной вершиной графа $\Lambda$, так как следующее звено ломаной $\sigma$ не входит в $\Lambda$. Таким образом, $c \in \partial \Lambda$ и к вершине $c$ в $\Lambda$ примыкает ребро графа $\Gamma_{2}$, что противоречит условию. Следовательно, утверждение $1^{0}$ выполнено.

Теорема доказана.

СлЕДСТВИЕ 1. Задача (1)-(6) вырождена тогда и только тогда, когда выполнено условие $1^{0}$ теоремы 3 (или, что то же самое, не выполнено условие теоремы 2).

СЛЕДСТВИЕ 2. Задача (1)-(6) вырождена тогда и толъко тогда, когда выполнено условие теоремь 1.

СЛЕДСТВИЕ 3. Задача (1)-(6) невырождена тогда и только тогда, когда выполнено условие теоремы 2. 
СЛЕДСТвИЕ 4. Задача (1)-(6) невырождена тогда и толъко тогда, когда не въполнено условие теоремы 1.

ТЕОРема 4. Задача (1)-(6) невырождена тогда и только тогда, когда $ә Г \neq \varnothing ~$ и выполнен принцип максимума.

ДокАзАтельство. Пусть задача (1)-(6) невырождена. Тогда из следствия 3 теоремы 3 имеем выполнение условия теоремы 2. Откуда следует $\partial \Gamma \neq \varnothing$.

Рассмотрим произвольное решение $u(\cdot)$ уравнения $\mathfrak{L} u=0$. Доопределим $u(x)$ до непрерывной функции на Г. Это можно сделать, так как в силу равномерной непрерывности и условий (3) пределы в каждой фиксированной внутренней вершине вдоль всех прилегающих ребер одинаковы. Кроме того, будем считать, что для каждой граничной вершины $a$ и любого ребра $\gamma$, прилежащего к вершине $a$, определено предельное значение $u_{\gamma}(a)$.

Предположим, что $M=\sup _{x \in \Gamma} u(x)$. Для некоторого ребра $\gamma$ и предельной точки $x_{0}$ этого ребра выполнено равенство $u_{\gamma}\left(x_{0}\right)=M$. Повторяя рассуждения теоремы 2 для точки $x_{0}$, получим, что значение $M$ функция $u(\cdot)$ достигает на границе графа.

Аналогичное рассуждение можно провести для $m=\inf _{x \in \Gamma} u(x)$. Таким образом, принцип максимума выполняется.

Пусть $\partial \Gamma \neq \varnothing$ и выполнен принцип максимума. Рассмотрим произвольное решение $u(\cdot)$ однородной задачи. Поскольку $\mathfrak{L} u=0$ и выполнен принцип максимума, то $M=\sup _{x \in \Gamma} u(x)$ и $m=\inf _{x \in \Gamma} u(x)$ достигаются на $\partial Г$. В силу условия (6) имеем $M=m=0$. Следовательно, $u(x) \equiv 0$ и задача (1)-(6) невырождена.

Теорема доказана.

\section{СПИСОК ЦИТИРОВАННОЙ ЛИТЕРАТУРЫ}

[1] Ю.В.Покорный, О.М. Пенкин, В.Л. Прядиев, А.В. Боровских, К. П. Лазарев, С. А. Шабров, Дифференциальные уравнения на геометрических графах, Физматлит, M., 2003.

[2] P. Kuchment, "Graph models for waves in then structures", Waves Random Media, 12 (2002), 1-24.

[3] Ю.В. Покорный, “О неосцилляции обыкновенных дифференциальных уравнений и неравенств на пространственных сетях", Дифферени. уравнения, 37:5 (2001), 661-671.

[4] О.М. Пенкин, Ю.В. Покорный, Е.Н. Провоторова, "Об одной векторной краевой задаче", Краевые задачи, Пермь, 1983, 64-70.

[5] О.М.Пенкин, Ю.В.Покорный, "О краевой задаче на графе”, Дифферени. уравнения, 24:4 (1988), 701-703.

[6] Ю. В. Покорный, О.М. Пенкин, “Теоремы Штурма для уравнений на графах”, Докл. AH CCCP, 309:6 (1989), 1306-1308.

[7] Ю. В. Покорный, О. М. Пенкин, "О теоремах сравнения для уравнений на графах", Дифферени. уравнения, 25:7 (1989), 1141-1150.

[8] Ю. В. Покорный, Е. Н. Провоторова, О.М.Пенкин, "О спектре некоторых векторных краевых задач", Вопросы качественной теории дифференииальных уравнений, Сб. научн. тр., Наука, Новосибирск, 1988, 109-113.

[9] J. E. Lagnese, G. Leugering, E. J. P. G. Schmidt, "Control of planar networks of Timoshenko beams", SIAM J. Control Optim., 31 (1993), 780-811.

[10] B. Dekoninck, S. Nicaise, "Spectre des réseaux de poutres", C.R. Acad. Sci. Paris, 326. Série 1 (1998), 1249-1254.

[11] B. Dekoninck, S. Nicaise, "The eigenvalue problem for networks of beams", Generalized Functions. Operator Theory and Dynamical Systems, Chapman and Hall Research in Math., 1999, 335-344. 
[12] F. Ali Mehmeti, B. Dekoninck, "Transient vibrations of planar networks of beams: interaction of flexion, transversal and longitudal waves", Partial Differential Equations on Multistructures, Lect. Notes Pure Appl. Math., 219, ed. F. Ali Mehmeti, J. von Below and S. Nicaise, 2001, 1-18.

[13] B. Dekoninck, "Control of network of Euler-Bernoulli beams", ESAIM-COCV, 4 (1999), $57-82$.

[14] J. E. Lagnese, G. Leugering, E. J.P. G. Schmidt, "Modeling of dynamic networks of thin thermoelastic beams", Math. Meth. Appl. Sci., 16 (1993), 327-358.

[15] A.V. Borovskikh, K.P. Lazarev, "Fourth-order differential equations on geometric graphs", J. Math. Sci., 119:6 (2004), 719-739.

[16] А. В. Боровских, Р. Мустафокулов, К. П. Лазарев, Ю. В. Покорный, “Об одном классе дифференциальных уравнений четвертого порядка на пространственной сети”, Докл. PAH, 345:6 (1995), 730-732.

[17] Ю. В. Покорный, Р. Мустафокулов, "О позитивной обратимости некоторых краевых задач для уравнений четвертого порядка", Дифферени. уравнения, 33:10 (1997), 13581365.

[18] Ю. В. Покорный, Р. Мустафокулов, "О положительности функции Грина линейных краевых задач для уравнений четвертого порядка на графе”, Изв. вузов. Математика, 441:2 (1999), 75-82.

[19] Ю. В. Покорный, К. П. Лазарев, "Некоторые осцилляционные теоремы для многоточечных задач", Дифферени. уравнения, 23:4 (1987), 658-670.

[20] К.П. Лазарев, О спектре некоторых негладких многоточечных задач, Дисс. ... к.ф.-м.н., ВГУ, Воронеж, 1988.

[21] Е.Н. Провоторова, "О векторных краевых задачах, порождаемых скалярным дифференциальным оператором", Дифферени. уравнения, 23:10 (1987), 1711-1715.

[22] Ю. В. Покорный, Т. В. Белоглазова, К. П. Лазарев, "Об одном классе разнопорядковых обыкновенных дифференциальных уравнений на графе", Матем. заметки, 73:3 (2003), 469-470.

\section{К. П. Лазарев}

Воронежский государственный университет

E-mail: lazarev@kvm.vsu.ru

\section{Т. В. Белоглазова}

Воронежский военный авиационный инженерный институт

E-mail: Sprite2000@mail.ru
Поступила в редакцию 23.12.2004

Исправленный вариант 05.09.2005 